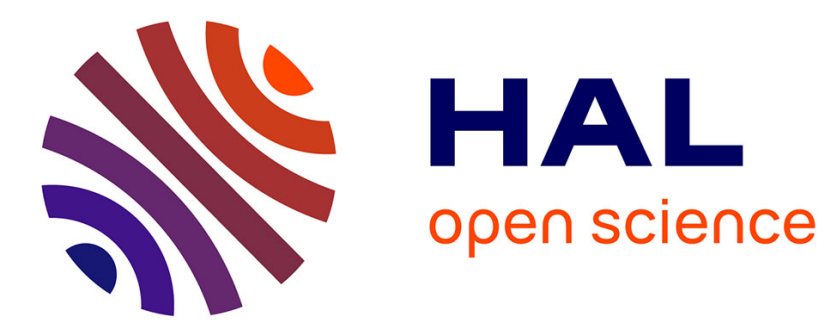

\title{
Crossover from impurity-controlled to granular superconductivity in (TMTSF)_2ClO_4
}

Shingo Yonezawa, Claire Marrache-Kikuchi, Klaus Bechgaard, Denis Jérome

\section{To cite this version:}

Shingo Yonezawa, Claire Marrache-Kikuchi, Klaus Bechgaard, Denis Jérome. Crossover from impurity-controlled to granular superconductivity in (TMTSF)_2ClO_4. Physical Review B, 2018, 97 (1), 10.1103/physrevb.97.014521 . hal-01964807

\section{HAL Id: hal-01964807 \\ https://hal.science/hal-01964807}

Submitted on 23 Dec 2018

HAL is a multi-disciplinary open access archive for the deposit and dissemination of scientific research documents, whether they are published or not. The documents may come from teaching and research institutions in France or abroad, or from public or private research centers.
L'archive ouverte pluridisciplinaire HAL, est destinée au dépôt et à la diffusion de documents scientifiques de niveau recherche, publiés ou non, émanant des établissements d'enseignement et de recherche français ou étrangers, des laboratoires publics ou privés. 


\title{
Crossover from impurity-controlled to granular superconductivity in (TMTSF) $)_{2} \mathrm{ClO}_{4}$
}

\author{
Shingo Yonezawa, ${ }^{1, *}$ Claire A. Marrache-Kikuchi, ${ }^{2}$ Klaus Bechgaard, ${ }^{3}$ and Denis Jérome ${ }^{4}$ \\ ${ }^{1}$ Department of Physics, Graduate School of Science, Kyoto University, Kyoto 606-8502, Japan \\ ${ }^{2}$ CSNSM, Université Paris-Sud, CNRS/IN2P3, 91405 Orsay, France \\ ${ }^{3}$ Department of Chemistry, Oersted Institute, Universitetsparken 5, 2100 Copenhagen, Denmark \\ ${ }^{4}$ Laboratoire de Physique des Solides (UMR 8502), Université Paris-Sud, 91405 Orsay, France
}

(Received 6 September 2017; published 23 January 2018)

\begin{abstract}
Using a proper cooling procedure, a controllable amount of nonmagnetic structural disorder can be introduced at low temperature in $(\mathrm{TMTSF})_{2} \mathrm{ClO}_{4}$. Here we performed simultaneous measurements of transport and magnetic properties of (TMTSF) ${ }_{2} \mathrm{ClO}_{4}$ in its normal and superconducting states, while finely covering three orders of magnitude of the cooling rate around the anion ordering temperature. Our result reveals, with increasing density of disorder, the existence of a crossover between homogeneous defect-controlled $d$-wave superconductivity and granular superconductivity. At slow cooling rates, with small amount of disorder, the evolution of superconducting properties is well described with the Abrikosov-Gorkov theory, providing further confirmation of non- $s$-wave pairing in this compound. In contrast, at fast cooling rates, zero resistance and diamagnetic shielding are achieved through a randomly distributed network of superconducting puddles embedded in a normal conducting background and interconnected by proximity effect coupling. The temperature dependence of the ac complex susceptibility reveals features typical for a network of granular superconductors. This makes (TMTSF) ${ }_{2} \mathrm{ClO}_{4}$ a model system for granular superconductivity where the grain size and their concentration are tunable within the same sample.
\end{abstract}

DOI: 10.1103/PhysRevB.97.014521

\section{INTRODUCTION}

Most of the recently discovered so-called "unconventional superconductors," such as the quasi-one-dimensional (Q1D) organic superconductors [1,2], copper oxides [3], two-dimensional organic superconductors based on bisethylenedithio-tetrathiafulvalene (BEDT-TTF) or related molecules [4-6], and the layered iron pnictides [7], share a unifying property: they exhibit a general phase diagram where superconductivity has a common border with a magnetically ordered phase. For such superconductors, the stability of superconductivity against nonmagnetic defects provides important information toward understanding of superconducting (SC) properties such as the pairing symmetry. As a particular example, in the ruthenate superconductor $\mathrm{Sr}_{2} \mathrm{RuO}_{4}$ [8], a strong reduction of $T_{\mathrm{c}}$ by a small amount of nonmagnetic impurity lends support for triplet $p$-wave pairing [9]. Since the nonmagnetic impurity effect is still actively debated for systems such as multiband superconductors [10] and topological superconductors [11,12], a detailed and controlled investigation of the impurity effect is an important subject.

Q1D organic superconductors are textbook examples of unconventional superconductivity, with the competition between density wave (insulating) and pairing (superconducting) orderings, namely the Peierls and Cooper divergences [13-15]. Reviews can be found in Refs. [16-18]. In the case of $(\mathrm{TMTSF})_{2} \mathrm{PF}_{6}$, a prototype of the (TMTSF $)_{2} X$ family, where TMTSF is the tetramethyl-tetrafulvalene electron-donor molecule and $X$ is a monovalent anion, the balance between

\footnotetext{
*yonezawa@scphys.kyoto-u.ac.jp
}

the two possible ground states is controlled by the magnitude of the kinetic coupling between molecular stacks [19,20]. Hence, the interplay between Peierls and Cooper channels can be controlled by an applied hydrostatic pressure: Under low pressures, a SDW ground state is stable [1,16] due to the good nesting of the warped Fermi surfaces, whereas superconductivity is stable above $\approx 9$ kbar when antinesting terms become dominant in their dispersion relation.

The only ambient-pressure superconductor in the (TMTSF $)_{2} X$ family, namely (TMTSF) $)_{2} \mathrm{ClO}_{4}$, was found shortly after the discovery of (TMTSF) ${ }_{2} \mathrm{PF}_{6}$ [2]. The SC order parameter of the (TMTSF $)_{2} X$ family has been thoroughly investigated mainly on (TMTSF) ${ }_{2} \mathrm{ClO}_{4}$ [21-27]. Although the debate is not fully settled yet, one of the most plausible scenarios is the magnetic-fluctuation driven nodal $d$-wave-like pairing, as strongly demonstrated by the field-angle-dependent heat-capacity measurements [26,28]. Such a possibility has been indeed theoretically proposed even from early days [29-33]. We note that, within this scenario, the gap nodes are accompanied by nontrivial topological features, as in the case of the nodes in $d$-wave superconductors [34]. Thus (TMTSF $)_{2} X$ is a candidate for topological nodal superconductors [35], which are of interest in the context of recently developing topological materials science.

The impurity effect on the (TMTSF) ${ }_{2} X$ family has been investigated for a long time. The remarkable sensitivity of superconductivity to irradiation $[36,37]$ has first been considered as a signature for triplet pairing [38]. However, defects introduced by irradiation in a controlled way [39] can often be magnetic [40]. Hence, the suppression of superconductivity by irradiation-induced defects must be taken with care since local magnetic impurities can act as strong pair breakers even on 
$s$-wave superconductors. A softer way to introduce nonmagnetic impurities in the (TMTSF $)_{2} X$ series is to produce a solid solution of isoelectronic anions. The solid solution procedure of (TMTSF) ${ }_{2} \mathrm{ClO}_{4}$, replacing with the tetrahedral anion $\mathrm{ClO}_{4}$ with $\mathrm{ReO}_{4}$, leads to a suppression of $T_{\mathrm{c}}$ due to genuine nonmagnetic impurities [23,41]. These results provided strong evidence for the realization of a non- $s$-wave pairing state in $(\mathrm{TMTSF})_{2} X$.

The ambient-pressure superconductor (TMTSF) ${ }_{2} \mathrm{ClO}_{4}$ provides another unique means to control its unconventional superconductivity by disorder. This material exhibits superconductivity below $T_{\mathrm{c}} \sim 1.3 \mathrm{~K}$ if the sample is cooled slowly $[2,42,43]$. However, if the sample is cooled fast enough in the vicinity of $25 \mathrm{~K}$, (TMTSF) ${ }_{2} \mathrm{ClO}_{4}$ undergoes a spin-densitywave (SDW) transition towards an insulating ground state below 4-5 K [44-46]. This significant feature of (TMTSF) ${ }_{2} \mathrm{ClO}_{4}$ is actually related to the noncentrosymmetric nature of the tetrahedral $\mathrm{ClO}_{4}$ anion located on the inversion centers of the full structure. At high temperature, the thermal motion of the $\mathrm{ClO}_{4}$ orientation makes it possible to preserve inversion symmetry on average since $\mathrm{ClO}_{4}$ occupies randomly one or the other inversion-symmetry-related orientations. The structural disorder between two possible orientations no longer persists at low temperatures below $T_{\mathrm{AO}}=24 \mathrm{~K}$, because an entropy gain due to the reduction of degrees of freedom triggers the anion ordering below this temperature.

The ordering transition has been studied by several techniques. In particular, diffuse x-ray work has shown that, while $\mathrm{ClO}_{4}$ anions adopt a uniform orientation along $a$ and $c$ axes, they alternate along $b$ [47] when the crystal is cooled very slowly through $T_{\mathrm{AO}}$, to reach the so-called relaxed state $[44,48]$. Since the dynamics of the anion orientation was shown to be slow $[44,49,50]$, fast cooling (i.e., cooling much faster than $17 \mathrm{~K} / \mathrm{min}$ by quenching) enables us to retain anion disorder even below $T_{\mathrm{AO}}$ in a metastable state with a single pair of Fermi-surface sheets resembling closely that of (TMTSF) ${ }_{2} \mathrm{PF}_{6}$. Consequently, the good nesting of the single-pair Fermi surfaces at $\pm k_{\mathrm{F}}$ for the quenched material stabilizes the insulating SDW state. In contrast, with the slow enough cooling rate, the alternating order of the anions along the $b^{\prime}$ axis leads to a folding of the Fermi surface, opening a gap at $\pm b^{*} / 4$ and resulting in doubling of the Fermi-surface sheets. This folding suppresses the SDW state by disturbing the Fermi-surface nesting, instead allowing superconductivity to appear below $1.2-1.3 \mathrm{~K}$.

Although the two extreme situations, namely the relaxed and quenched states, with SC and SDW ground states respectively, have been fairly extensively studied from the early days, only limited studies have been published for the intermediate cooling-rate regime, where superconductivity is moderately suppressed [23,51,52]. High-resolution X-ray investigations [53-55] have shown that samples in the intermediate coolingrate regime exhibit a peculiar anion ordering, in which domains with ordered anions of finite size are embedded in a disordered background. In the regime up to $5 \mathrm{~K} / \mathrm{min}$, high-resolution $\mathrm{X}$-ray-diffraction measurements have determined both the volume fraction of ordered anions and the average size of ordered domains [55].

Here, we report on the resistivity $\rho_{c^{*}}$ along the least conducting $c^{*}$ axis [56] simultaneously measured with the ac susceptibility $\chi_{\text {ac }}$ under carefully controlled cooling across $T_{\mathrm{AO}}$, in order to address the changes of properties of the normal and SC phases by the increase of disorder. With the experimental settings especially designed for the present investigation, we explored the cooling rate around $T_{\mathrm{AO}}$ from 0.02 to $18 \mathrm{~K} / \mathrm{min}$ with fine steps, nearly covering three orders of magnitude. We believe that the present study is one of the most careful impurity-effect studies in any unconventional superconductor, because we control the amount of the defects in identical samples, in a reversible way, and with very fine intervals. From our transport and magnetic measurements, we reveal that the intermediate cooling-rate regime of (TMTSF) ${ }_{2} \mathrm{ClO}_{4}$ cannot be considered as a state exhibiting an average anion order/disorder evolving according to the cooling rate, but instead as a state comprising a cooling-rate-dependent volume fraction of well-ordered domains with the rest of the volume occupied by disordered anions. We also study the evolution of the onset $T_{\mathrm{c}}$ of $\chi_{\mathrm{ac}}(T)$ and that of $\rho_{c^{*}}(T)$. We note that $T_{\mathrm{c}}$ under fast cooling has been studied using a time-dependent Ginzburg-Landau theory [57]. However, this earlier study was only based on transport properties.

\section{EXPERIMENT}

For the present study, two single crystals of (TMTSF) ${ }_{2} \mathrm{ClO}_{4}$ from the same batch grown with an electrocrystallization method have been used. Simultaneous transport and susceptibility measurements have been performed on one sample (no. 1 ; with the size of $\approx 2.4 \times 0.7 \times 0.1 \mathrm{~mm}^{3}$ ). The results thus obtained will constitute the core of the paper. With a slightly different setup with an additional thermometer directly attached beneath the sample, the ac susceptibility was measured for another sample (no. 2; with the size of $\approx 1.5 \times 0.4 \times 0.1 \mathrm{~mm}^{3}$ ). Results for sample no. 2 and details of the measurement setup will be given in the Supplemental Material [58].

For both experiments, the samples were cooled down with a commercial cryostat (Quantum Design; Physical Property Measurement System (PPMS)) with an adiabatic demagnetization refrigerator (ADR) option. In order to control the disorder in the $\mathrm{ClO}_{4}$ orientation within a sample, we first raised the temperature to $50 \mathrm{~K}$ and kept it for more than $1 \mathrm{~h}$ to fully randomize the anion orientations. The sample was first cooled in a controlled way down to $10 \mathrm{~K}$ using the temperature controller of the PPMS. After further cooling down to $1.8 \mathrm{~K}$, the temperature was kept at $1.8 \mathrm{~K}$ and the dc magnetic field of $2.5 \mathrm{~T}$ applied. The sample chamber was then evacuated with a turbomolecular pump to achieve high-vacuum adiabatic condition. Then the magnetic field was turned off to reach $\sim 0.1$ $\mathrm{K}$, and the transport or magnetic data were collected during warming. See Fig. S2 of the Supplemental Material [58] as well as Fig. 8 of Ref. [59] for details of the cooling sequence of the ADR option. We should comment here that the sample was always in the field-cooled condition although all measurements were performed in zero dc field.

The resistivity of the sample was measured with the resistivity option of the PPMS. The current of $10 \mu \mathrm{A}(1 \mathrm{~mA}$ for measurements at high temperatures) along the $c^{*}$-axis direction was reversed to cancel the thermoelectric voltage. The ac susceptibility was measured with an ac field of $\sim 0.17-$-Oe rms 
at $3011 \mathrm{~Hz}$ (sample no. 1) or $887 \mathrm{~Hz}$ (sample no. 2) parallel to the $c^{*}$ axis, using a conventional mutual inductance method with a miniature susceptometer [59] fitting into the ADR option of the PPMS. The output signal of the susceptometer was measured using a lock-in amplifier (Stanford Research Systems, SR830). To correct a small mixing of the real and imaginary parts in the lock-in amplifier signal, an additional phase factor $\theta$ is adopted: $\Delta \chi_{\mathrm{ac}}^{\prime} \propto \Delta V_{y} \cos (\theta)+\Delta V_{x} \sin (\theta)$ and $\Delta \chi_{\mathrm{ac}}^{\prime \prime} \propto \Delta V_{x} \cos (\theta)-\Delta V_{y} \sin (\theta)$, where $\Delta V_{x}$ and $\Delta V_{y}$ are changes in the reading of the lock-in amplifier. The value of $\theta$ is chosen as $\theta=1.5^{\circ}$ and $8.5^{\circ}$ for samples no. 1 and no. 2 , respectively, so that $\Delta \chi_{\mathrm{ac}}^{\prime \prime}=0$ for $T>T_{\mathrm{c}}$ and $T \rightarrow 0$ for the $0.020-\mathrm{K} / \mathrm{min}$ data.

\section{EXPERIMENTAL DATA}

Figure 1(a) displays the temperature dependence of $\rho_{c^{*}}$ up to $50 \mathrm{~K}$ upon cooling at $0.02 \mathrm{~K} / \mathrm{min}$. A clear anomaly at $T_{\mathrm{AO}}=$ $24.5 \mathrm{~K}$ is observed. By comparing the fittings using polynomial functions to the $\rho_{c^{*}}(T)$ data above $25 \mathrm{~K}$ and below $12 \mathrm{~K}$, we can notice that $\rho_{c^{*}}(T)$ jumps by $\Delta \rho_{c^{*}} \approx 0.23 \Omega \mathrm{cm}$, as indicated with the red arrow. This resistance drop reflects the decrease of the elastic scattering due to anion ordering.

Figures 1(b)-1(d) show the evolutions of the imaginary and real parts of the ac susceptibility $\left[\Delta \chi_{\mathrm{ac}}^{\prime \prime}(T)\right.$ and $\Delta \chi_{\mathrm{ac}}^{\prime}(T)$ respectively] and of $\rho_{c^{*}}$ with the cooling rate. All three quantities show that the sample cooled down at $0.02 \mathrm{~K} / \mathrm{min}$ exhibits superconductivity below $T_{\mathrm{c}}(0.02 \mathrm{~K} / \mathrm{min}) \sim 1.3 \mathrm{~K}$. The superconducting features were progressively weakened upon increasing the cooling rate. We comment that the small upturn in $\Delta \chi_{\mathrm{ac}}^{\prime \prime}(T)$ and $\Delta \chi_{\mathrm{ac}}^{\prime}(T)$ below $0.3 \mathrm{~K}$ is probably extrinsic, since such behavior was not observed in another sample (sample no. 2; see the Supplemental Material [58]). Perhaps the upturn could be attributed to the small background signal originating from the attached electrical leads and silver paint used for the resistivity measurements.

Based on these raw data, the cooling-rate dependence of various superconducting properties can be examined: $T_{\mathrm{c} \chi}$ is defined from the onset of $\Delta \chi_{\mathrm{ac}}^{\prime}(T)$ and $T_{\mathrm{c} \rho}$ by the peak in the second derivative of $\rho_{c^{*}}(T)$. The zero-resistivity temperature $T_{\mathrm{c}, \rho=0}$ is the temperature where a linear extrapolation of the steep transition in $\rho_{c^{*}}(T)$ reaches zero. We found that $T_{\mathrm{c}, \rho=0}$ is approximately equal to $T_{\mathrm{c} x}$. The evolutions of the different $T_{\mathrm{c}}$ 's with the cooling rate are given in Fig. 2(a). The small difference in $T_{\mathrm{c} \chi}$ for samples no. 1 and no. 2 can be attributed to different ordering configurations which can exist even in samples of the same batch. As noticed in previous X-ray studies [49], no perfect long-range order of anions exists even in the slowest cooled samples. Let us moreover note that $T_{\mathrm{c} x}$ is not a thermodynamic quantity and, as we will later see, derives from a specific phase distribution within the sample.

Next, we focus on the change in the shielded volume fraction. Since the samples cooled at rates smaller than $0.02 \mathrm{~K} / \mathrm{min}$ are fully in the relaxed-state regime and are expected to display perfect diamagnetism at low temperatures, we will consider this cooling rate as a reference. Figure 2(b) displays the cooling-rate dependence of the relative SC shielded volume fraction $v_{\text {shield }}$, which is obtained from the zero-temperature extrapolation of $\Delta \chi_{\mathrm{ac}}^{\prime}(T)$ divided by its value for $0.02 \mathrm{~K} / \mathrm{min}$ : $v_{\text {shield }} \equiv\left|\Delta \chi_{\text {ac }}^{\prime}(T \rightarrow 0)\right| /\left|\Delta \chi_{\text {ac }}^{\prime}(T \rightarrow 0,0.02 \mathrm{~K} / \mathrm{min})\right|$.

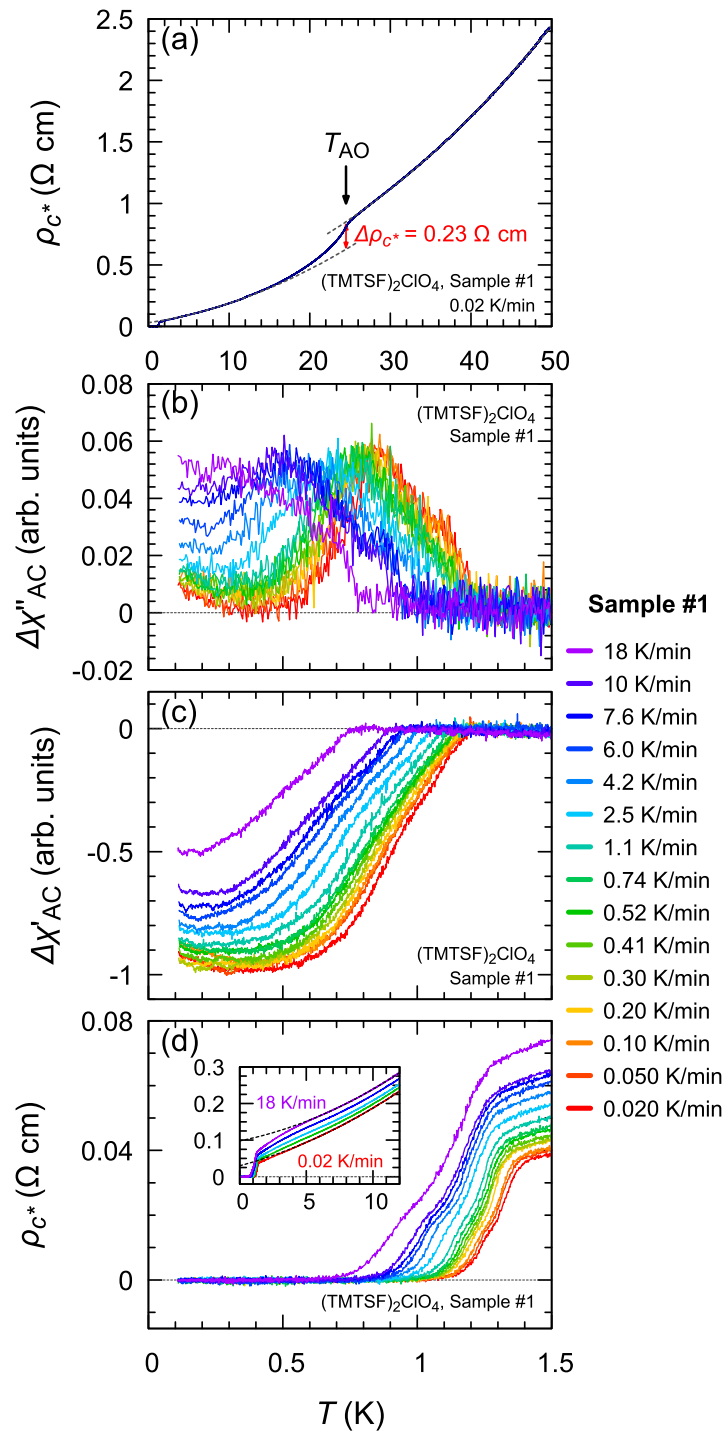

FIG. 1. Normal and superconducting properties of (TMTSF) ${ }_{2} \mathrm{ClO}_{4}$ for sample no. 1 at various cooling rates. (a) Interlayer resistivity $\rho_{c^{*}}$ vs $T$ at $0.020 \mathrm{~K} / \mathrm{min}$ showing the drop of the elastic resistivity $\Delta \rho_{c^{*}}=0.23 \Omega \mathrm{cm}$ at $T_{\mathrm{AO}}$ as indicated by the red arrow. (b) Change in the imaginary part of the ac susceptibility. (c) Change in the real part of the ac susceptibility. (d) $\rho_{c^{*}}(T)$ simultaneously measured with the data in (b) and (c). The inset presents $\rho_{c^{*}}(T)$ on a broader temperature range for $0.020,0.52,2.5$, 7.6 , and $18 \mathrm{~K} / \mathrm{min}$. For the 0.020 - and $18-\mathrm{K} / \mathrm{min}$ data, the results of the fitting, performed using the procedure explained in the text, are presented with the broken curves.

The zero-temperature extrapolation of $\Delta \chi_{\mathrm{ac}}^{\prime \prime}$ averaged on the $0.1-0.3-\mathrm{K}$ range is also given in Fig. 2(d). We will discuss the meaning of this quantity in Sec. IV C.

The normal-state residual resistivity $\rho_{c^{*} 0}$ presented in Fig. 2(c) is obtained by fitting the relation $\rho_{c^{*}}(T)=\rho_{c^{*} 0}+$ $A T+B T^{2}$ to the data between 6 and $12 \mathrm{~K}$ [see the inset of Fig. 1(d), as well as Fig. 1(a)]. We chose this fitting range to avoid the influence of the downturn of $\rho_{c^{*}}(T)$, quite prominent below $5 \mathrm{~K}$ especially in fast cooled samples. We note that the low-temperature downturn in $\rho_{c^{*}}(T)$ is ascribed to the sliding of SDW fluctuations [60] and will 


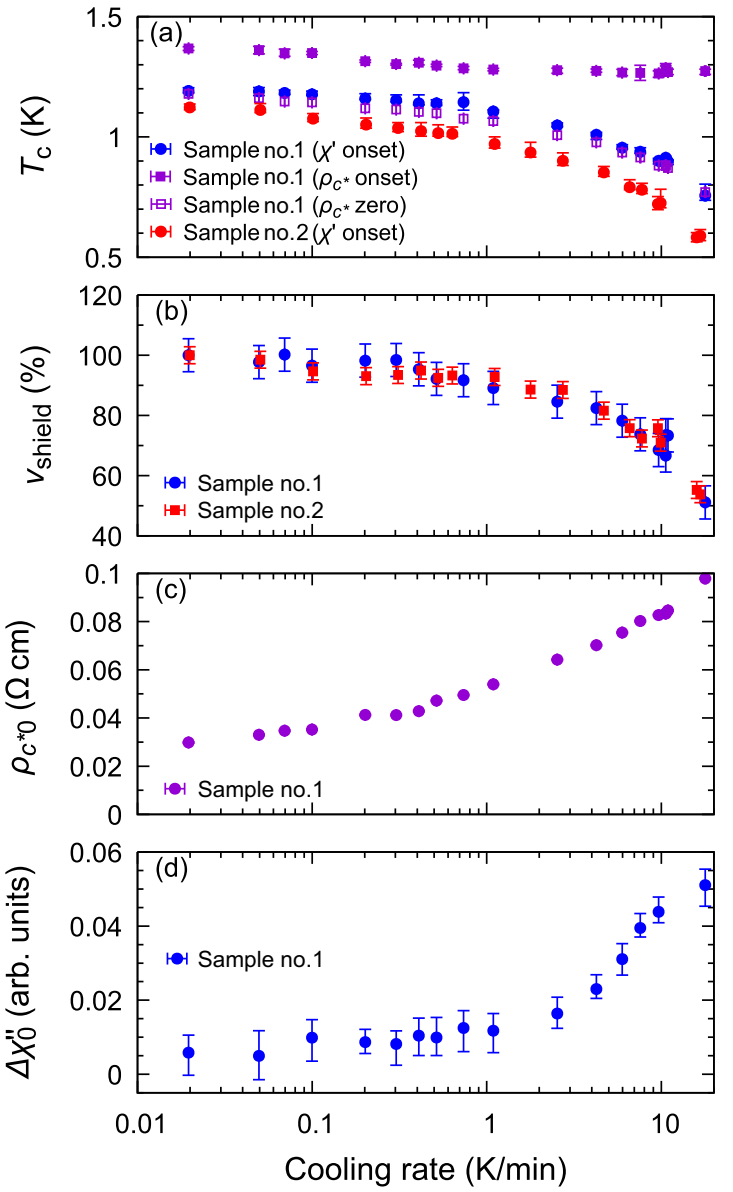

FIG. 2. Cooling-rate dependence of superconducting properties for sample no. 1, and its comparison to that of sample no. 2. (a) Cooling-rate dependence of the various critical temperatures: the blue circles correspond to $T_{\mathrm{c} \chi}$, the purple closed squares to $T_{\mathrm{c} \rho}$, and the purple open squares to $T_{\mathrm{c}, \rho=0}$. The red circles indicate $T_{\mathrm{cx}}$ for sample no. 2. (b) Cooling-rate dependence of the relative shielded volume fraction $v_{\text {shield }}$ for samples no. 1 (blue) and no. 2 (red). (c) Cooling-rate dependence of the residual resistivity $\rho_{c^{*} 0}$. (d) Low-temperature dissipation, as measured by $\Delta \chi_{\text {ac }}^{\prime \prime}$ for sample no. 1 . The $\Delta \chi_{\mathrm{ac}}^{\prime \prime}$ data have been averaged in the temperature range $0.1 \mathrm{~K}<T<0.3 \mathrm{~K}$.

be examined in detail in a forthcoming publication. Such a fitting procedure is justified by previous extensive investigations ascribing scattering against spin fluctuations as the source of the dominant linear temperature dependence of the resistivity [61-63].

Systematic decreases of all characteristic temperatures are observed with increasing cooling rates, as expected when disorder is increased. However, although the decrease of $T_{\mathrm{c} \rho}$ levels off at a value of $\approx 1.25-1.30 \mathrm{~K}$ above $1 \mathrm{~K} / \mathrm{min}, T_{\mathrm{c} x}$ reveals a faster decrease above the same cooling rate. The shielded volume fraction for $T \rightarrow 0$ steadily decreases with increasing cooling rate. Concomitantly, we notice an acceleration in the decrease of $v_{\text {shield }}$ above $1 \mathrm{~K} / \mathrm{min}$. No more than $50 \%$ of the sample volume is shielded at $18 \mathrm{~K} / \mathrm{min}$.

Explaining the difference in behavior between $T_{\mathrm{c} \rho}$ and $T_{\mathrm{c} x}$ is the major issue of the present work and will underline the interpretation proposed in Sec. IV C.
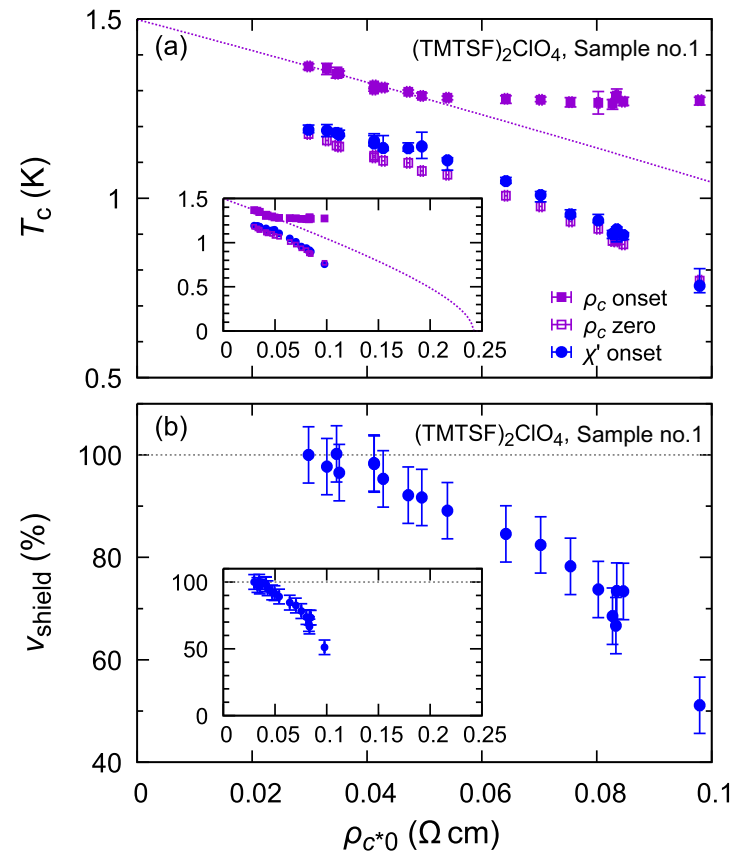

FIG. 3. Dependence of $T_{\mathrm{c}}$ 's and relative shielded volume fraction $v_{\text {shield }}$ on $\rho_{c^{*} 0}$ for sample no. 1. (a) $T_{\mathrm{c} \rho}$ as a function of $\rho_{c^{*} 0}$, compared with $T_{\mathrm{c}, \rho=0}$ and $T_{\mathrm{c} \chi}$. The dotted curve presents results of fittings of $T_{\mathrm{c} \rho}$ with the AG formula [Eq. (2)]. The fitting has been performed in the range $0<\rho_{c^{*} 0}<0.05 \Omega \mathrm{cm}$. The inset shows the same data in a wider range. (b) $v_{\text {shield }}$ as a function of $\rho_{c^{*} 0}$. The inset shows the same data in a wider range.

\section{DISCUSSION}

\section{A. Determination of the anion-ordered volume}

So far, quantities such as $T_{\mathrm{c}}, \rho_{c^{*} 0}$, or $v_{\text {shield }}$ were plotted against the cooling rate in Fig. 2. Since the amount of impurities introduced by the cooling may depend on samples, as well as on the details of the cooling procedure, the physical picture is better conveyed when representing $T_{\mathrm{c}}$ and $v_{\text {shield }}$ as functions of the residual resistivity, as shown in Figs. 3(a) and 3(b). These figures reveal two regimes: below around $\rho_{c^{\star} 0} \approx 0.05 \Omega \mathrm{cm}$ (corresponding to the cooling rate of $\approx 1 \mathrm{~K} / \mathrm{min}$ ), $T_{\mathrm{c}}$, by all definitions, exhibits a steady decrease with increasing cooling rate whereas $v_{\text {shield }}$ stays $\sim 100 \%$. However, above this threshold, $T_{\mathrm{c} \rho}$ becomes nearly independent of the cooling rate, whereas $v_{\text {shield }}$ clearly decreases. This fact suggests a possible change in the sample behavior. Particularly, the substantial decrease in $v_{\text {shield }}$ indicates that rapid cooling is no longer equivalent to creating local defects, and instead promotes the formation of clusters of disordered regions.

Expecting the picture of local defects to be no longer adequate at large cooling rates, it is important to derive the actual volume fraction in which bulk superconductivity develops since, in an inhomogeneous superconductor, it could be smaller than the shielded volume.

This volume fraction can be reached viewing the normalstate sample as a two-component composite conductor with resistivities $\rho_{\text {Min }}$ and $\rho_{\text {Max }}$ for ordered and disordered regions respectively. Such a problem of the mixture between two conductors of different conductivities is formally identical [64] 


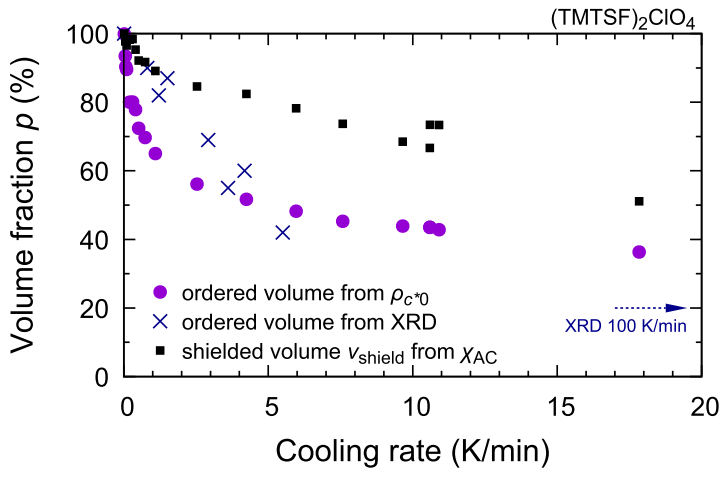

FIG. 4. Volume fraction of the anion-ordered domain derived from resistivity ( $p$, obtained from the effective-medium theory in this work, purple circles) and from high-resolution $\mathrm{x}$ rays ( $v_{\mathrm{XRD}}$, from Ref. [55], blue crosses), compared with the shielded fraction evaluated from the ac susceptibility ( $v_{\text {shield }}$, black squares). The broken arrow shows the estimate for the ordered volume from $\mathrm{x}$-ray measurements at $100 \mathrm{~K} / \mathrm{min}$ [53].

to the effective dielectric permeability of a two-component dielectric medium treated by Landau and Lifshitz [65]. The mixed conductor can thus be described at the lowest order by a three-dimensional (3D) effective-medium theory with an effective conductivity $\sigma_{\text {eff }}=1 / \rho_{\text {eff }}$, which can be derived as

$$
1 / \rho_{\text {eff }}=\left[p\left(1 / \rho_{\text {Min }}\right)^{1 / 3}+(1-p)\left(1 / \rho_{\text {Max }}\right)^{1 / 3}\right]^{3},
$$

where $p$ is the volume fraction of the anion-ordered domain. The resistivity in the ordered region $\rho_{\text {Min }}$ is given by $\rho_{c^{*} 0}$ for $0.02 \mathrm{~K} / \mathrm{min}$, based on the assumption that the system at the lowest cooling rate provides $\rho_{\text {Min. }}$. The data in Fig. 1 lead to $\rho_{\text {Min }}=0.030 \Omega \mathrm{cm}$. For the disordered resistivity $\rho_{\text {Max }}$, the change in the elastic contribution $\Delta \rho_{c^{*}}$ across $T_{\mathrm{AO}}$, coming from the frozen anion disorder, must be added to $\rho_{\text {Min }}$, i.e., $\rho_{\text {Max }}=\rho_{\text {Min }}+\Delta \rho_{c^{*}}=0.26 \Omega \mathrm{cm}[66]$.

Figure 4 compares the ordered volume fraction $p$ obtained from Eq. (1) with the shielded volume $v_{\text {shield }}$ and with the ordered volume fraction $v_{\text {XRD }}$ derived from x-ray measurements (Ref. [55]). Considering the uncertainties in the x-ray determination, there is a fair agreement between $v_{\text {XRD }}$ and $p$, particularly in the fast-cooling regime. $p$ obtained at high cooling rates is also of the same order as $v_{\mathrm{XRD}}$ at even higher disorder (broken arrow on Fig. 4). Both $p$ and $v_{\mathrm{XRD}}$ reflect the size of the ordered domains, which can reasonably be assumed to be SC. In contrast, $v_{\text {shield }}$ measures the shielded volume fraction. Obviously, $p$ and $v_{\mathrm{XRD}}$ are always smaller than $v_{\text {shield }}$ in particular, at the highest cooling rate achieved in this study, $v_{\text {shield }}$ is of about $50 \%$, whereas $p$ is reduced down to $36 \%$. This fact can be seen as the signature of persistent shielding currents penetrating non-SC regions. The derivation of the relation between the ordered volume fraction and the cooling rate (Fig. 4) enables us to show how $T_{\mathrm{c} \rho}$ and the residual resistivity evolve against the disordered volume fraction; see Fig. 5. This figure reveals the occurrence of a regime change for both $T_{\mathrm{c} \rho}$ and $\rho_{c^{*} 0}$ around $30 \%$ disordered volume fraction. This feature will be studied more extensively in the following sections.
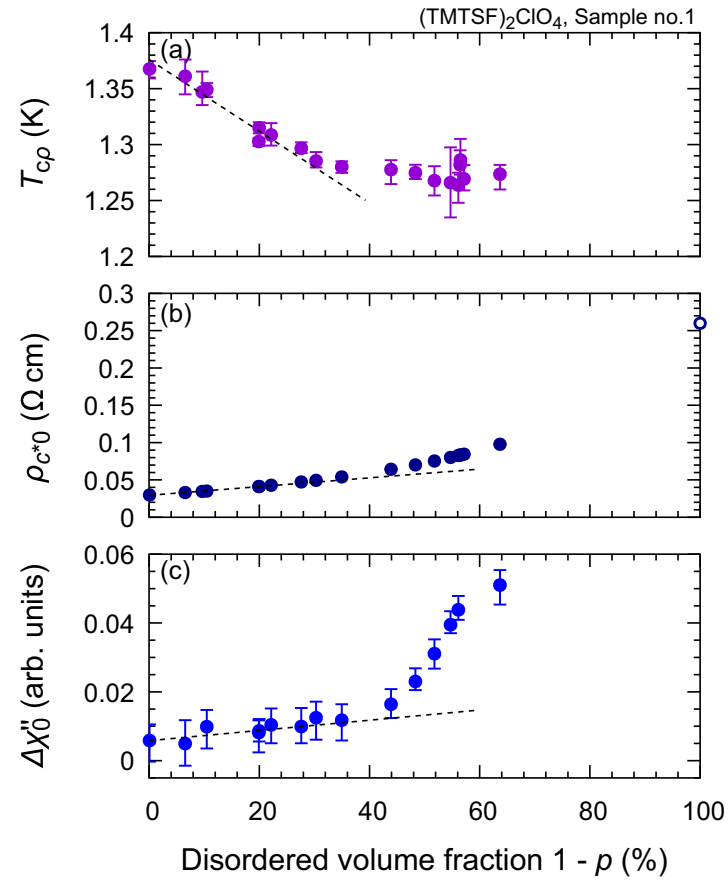

FIG. 5. (a) $T_{\mathrm{c} \rho}$, (b) $\rho_{c^{*} 0}$, and (c) low-temperature dissipation as measured by $\Delta \chi_{\mathrm{ac}}^{\prime \prime}$, plotted against the disordered volume fraction $1-p$ derived through Eq. (1). Deviation from the linear dependence (broken lines; fitted in the range $1-p<20 \%$ in all panels) occurs when the first finite disordered cluster emerges. At complete disorder (not accessible before the onset of the SDW ground state above $18 \mathrm{~K} / \mathrm{min}$ ), the resistivity would amount to $\rho_{\text {Max }}=0.26 \Omega \mathrm{cm}$, as indicated by the open circle. The $\Delta \chi_{\mathrm{ac}}^{\prime \prime}$ data in (c) have been averaged in the temperature range $0.1 \mathrm{~K}<T<0.3 \mathrm{~K}$.

\section{B. Local defect regime}

Let us call the low cooling rate regime $(\leqslant 1 \mathrm{~K} / \mathrm{min})$ "regime I," where both $T_{\mathrm{c} \rho}$ and $T_{\mathrm{c} \chi}$ decrease linearly with $\rho_{c^{*} 0}$ [i.e., in the range $\rho_{c^{*} 0} \leqslant 0.05 \Omega \mathrm{cm}$; see Fig. 3(a)]. In this regime, $\rho_{c^{*} 0}$ then is a good measure of disorder. Hence the usual analysis for the dependence of $T_{\mathrm{c}}$ on nonmagnetic disorder in $d$-wave superconductors $[67,68]$ can be undertaken.

The Abrikosov-Gorkov theory [69] (AG) extended to nonmagnetic impurities in the case of non- $s$-wave superconductivity gives

$$
\ln \left(\frac{T_{\mathrm{c} 0}}{T_{\mathrm{c}}}\right)=\Psi\left(\frac{1}{2}+\frac{\alpha T_{\mathrm{c} 0}}{2 \pi T_{\mathrm{c}}}\right)-\Psi\left(\frac{1}{2}\right),
$$

where $\Psi(x)$ is the digamma function, $\alpha=\hbar / 2 \tau k_{B} T_{\mathrm{c} 0}$ is the depairing parameter, $\tau$ is the elastic scattering time, and $T_{\mathrm{c} 0}$ is the limit of $T_{\mathrm{c}}$ in the absence of any scattering (i.e., the limit of $\rho_{c^{*} 0} \rightarrow 0$ ). The fit of $T_{\mathrm{c}}$ derived from Eq. (2) to the data is presented in Fig. 3(a). Clearly, $T_{\mathrm{c} \rho}$ is well fitted in the slowcooling (small $\rho_{c^{*} 0}$ ) regime. This fact provides firm evidence that the superconductivity of (TMTSF) ${ }_{2} \mathrm{ClO}_{4}$ is of non- $s$-wave nature, and is consistent with previous works [23,24].

If driven by local defects only, the critical value of the residual resistivity for the suppression of superconductivity would read $\rho_{c^{*} 0 \mathrm{c}}=0.24 \Omega \mathrm{cm}$ from the fit to $T_{\mathrm{c} \rho}$ shown in the inset of Fig. 3(a). This value is practically identical to the critical resistivity $\rho_{c^{*} 0 \mathrm{c}}=0.23 \Omega \mathrm{cm}$ obtained for the 
suppression of superconductivity on the $\mathrm{ClO}_{4}$-side of the $(\mathrm{TMTSF})_{2}\left(\mathrm{ClO}_{4}\right)_{(1-x)}\left(\mathrm{ReO}_{4}\right)_{x}$ solid solution due to the presence of nonmagnetic $\mathrm{ReO}_{4}$ anions [28]. This gives additional confidence in the fact that, in regime $I$, the destruction of superconductivity can be explained by the effect of local defects.

The nature of disorder in the present situation of an anion misorientation is quite different from the disorder in a solid solution [24]. The anion misorientation leads to disorder of the local electronic structures. Hence, anions, to be active scattering centers, must form large enough clusters exhibiting a local electronic structure (the single sheet Fermi surface at $\pm k_{\mathrm{F}}$ ) differing appreciably from the double-sheet Fermisurface structure in the ordered regions [70]. Such a condition requires the broadening of the Fermi surface due to a finite size of clusters to be kept at minimum, leading in turn to be at least 50 unit cells along the $a$ axis [71].

The next task at hand is the derivation of the elastic mean free path for the best ordered sample. According to magnetotransport data, we have $9 \times 10^{-12} \mathrm{sec}$ as the value for $\tau$ at the liquid-helium temperature in the best-ordered situation [72], leading in turn to a corresponding mean free path $l_{0}$ along the $a$ axis [73] of $1620 \mathrm{~nm}$, taking the $a$-axis Fermi velocity of $v_{\mathrm{F} a}=1.8 \times 10^{7} \mathrm{~cm} \mathrm{~s}^{-1}$. Such an estimate for the mean free path is also in fair agreement with previous transport results, namely $l_{0}=1400 \mathrm{~nm}$ [74]. From the fitting of the AG theory to $T_{\mathrm{c} \rho}$ in regime $\mathrm{I}$, the average mean free path at the border of regime $\mathrm{I}$ is obtained as $l=972 \mathrm{~nm}$ at $1-p \sim 0.3$. Since $T_{\mathrm{c} \rho}$ is nearly constant beyond this regime, $l$ is reasonably assumed to be invariant, on average, for $1-p>0.3$.

Furthermore, as already explained, the extrapolation of the AG formalism [inset of Fig 3(a)] leads to the critical value $\rho_{c^{*} 0 \mathrm{c}}=0.24 \Omega \mathrm{cm}$, which is eight times larger than $\rho_{\text {Min }}$. Thus, the critical mean free path $l_{\mathrm{c}}$ corresponding to $\rho_{c^{*} 0 \mathrm{c}}$ is given by $l_{\mathrm{c}}=l_{0} / 8=202 \mathrm{~nm}$. This is in good agreement with the critical mean free path $l_{\mathrm{c}, \mathrm{d} \text {-wave }}=\pi \xi_{0} \sim 220 \mathrm{~nm}$ expected for the suppression of $d$-wave superconductivity [67], given the measured SC coherence length along the $a$ axis, $\xi_{0 a}=70 \mathrm{~nm}$ [75].

We comment here on the width of the superconducting transition. We claim that a broad distribution of the scattering length provides in turn a distribution of $T_{\mathrm{c}}$. Indeed, it has been reported that, even in the ordered phase achieved by very slow cooling, in which $\mathrm{ClO}_{4}$ anions are ordered in one of their two possible ordering patterns, the domain size is largely distributed [49]. This is also supported by the fact that (TMTSF) ${ }_{2} \mathrm{ClO}_{4}$ ordinarily exhibits a broader superconducting transition than the pressure-induced superconductor (TMTSF $)_{2} \mathrm{PF}_{6}$, which does not have any anion disorder because the $\mathrm{PF}_{6}$ anion is a centrosymmetric anion. Moreover, as analyzed above using the AG model, scattering centers in regime I are small-sized disordered clusters. It is reasonable to assume that these clusters nucleate on the walls between ordered domains, because disorder ordinarily tends to develop in the vicinity of preexisting disorder, namely the domain walls in our case. Then, the somewhat broad transition and the double-transition-like feature for our slowly cooled data (e.g., $0.02 \mathrm{~K} / \mathrm{min}$ ) can be attributed to such anion disorder remaining even under slow cooling. In addition, the sample can contain microcracks, which are often formed in organic crystals during cooling due to thermal-contraction strain, and such microcracks can also contribute to the broadness of the transition, by modulating $T_{\mathrm{c}}$ and/or by introducing additional weak-links within the sample. In sample no. 1, we indeed observed a few small jumps in the temperature dependence of resistivity from room temperature to the lowest temperature. Such jumps indicate formation of a small number of microcracks.

\section{Evidences for a granular behavior}

Let us now call the higher cooling-rate regime "regime II," for which $\rho_{c^{*} 0}>0.05 \Omega \mathrm{cm}$ and $1-p \geqslant 0.3$. In this regime, $T_{\mathrm{c} \rho}$ is nearly constant $\left(T_{c \rho}=1.27 \pm 0.03 \mathrm{~K}\right)$, whereas the width of the resistive transition, measured by $T_{\mathrm{c} \rho}-T_{\mathrm{c}, \rho=0}$, increases as the cooling rate increases and the system becomes more and more disordered. In this regime, the macroscopic SC coherence is weaker than what would be expected if superconductivity was controlled by local defects only.

In this section, we will demonstrate that this behavior can be interpreted as a consequence of granular superconductivity: SC puddles, consisting of an assembly of grains where $\mathrm{ClO}_{4}$ anions are ordered along one of the ordering patterns (updown-up-down ... or down-up-down-up ...; labeled as A or B, respectively) [49], are distributed within a metallic background consisting of anion-disordered regions.

The progressive establishment of superconductivity in regime II resembles in several respects the situations observed in the studies of superconductivity in 3D granular compounds [76] and in 2D hybrid superconductor-normalmetal-superconductor arrays [77-79]: superconductivity first appears in disconnected grains which couple via the proximity (or Josephson) effect at lower temperatures.

The system considered in this work differs from other granular materials [76] insofar as, as we will see, the averaged puddle size is larger than the SC coherence length (the averaged coherence length within the $a b$ plane is $\xi_{0}=$ $45 \mathrm{~nm}$; from the upper critical field measurements [75], $\xi$ were evaluated to be 70,30, and $2 \mathrm{~nm}$ along the $a, b$, and $c$ axes, respectively; and the averaged $\xi_{0}$ mentioned here is evaluated using the geometrical mean $\sqrt{\xi_{a} \xi_{b}}$ ), but is smaller than the penetration depth. Thus, bulk superconductivity is first established within each puddle while cooling. Moreover, there is a strong coupling between neighboring puddles since the interpuddle background (disordered regions) is metallic, albeit with a resistivity $\left(\rho_{\text {Max }}=0.26 \Omega \mathrm{cm}\right)$ about ten times larger than the puddle normal resistivity $\left(\rho_{\text {Min }}=0.030 \Omega \mathrm{cm}\right)$ (see Sec. IV A). Let us emphasize that, in the present system, the grain size is tunable and solely governed by the cooling rate using a single sample.

Thus, $T_{\mathrm{c} \rho}$ corresponds to the temperature at which superconductivity begins to appear within each puddle. It is governed by the puddle size $L$. Although the puddles probably have a broad distribution in size, an estimate of the upper limit of $L$ can be derived from the AG approach. Assuming that the electronic mean free path $l$ is limited by $L$, one then obtains $l \simeq L=970 \mathrm{~nm}$. Such a large puddle size implies that they are composed of an equal weight of domains $\mathrm{A}$ and $\mathrm{B}$, whose individual dimensions are of the order of $39 \mathrm{~nm}$ at $5 \mathrm{~K} / \mathrm{min}$, according to direct $\mathrm{X}$-ray measurements [55]. However, let us 
keep in mind that this estimated $L$ reflects the size of the largest puddles, which have the highest $T_{\mathrm{c} \rho}$.

A close look at the shape of the resistive transition shown in Fig. 1(d) reveals that it takes place in two stages (particularly visible on the run at $18 \mathrm{~K} / \mathrm{min}$ ). First, the resistance drops by a factor of about 2 before leveling off and giving rise to a second drop whose long tail ends at around $T_{\mathrm{c} \chi}$. The temperature width of the first transition $\Delta T_{1}$ is analogous to the one measured in regime I $\left[\left.\Delta T_{1} \simeq\left(T_{c \rho}-T_{c \chi}\right)\right|_{\text {regime I }} \simeq 0.2 \mathrm{~K}\right]$. It seems natural to ascribe the first drop to the onset of superconductivity in individual puddles, $\Delta T_{1}$ reflecting the distribution in the puddles size. The second resistive drop can then be attributed to the progressive establishment of SC coherence over the whole sample: the SC puddles progressively couple through proximity effect to form $\mathrm{SC}$ clusters. Let us also note that the value $1-p=0.3$, at which regime II begins, is actually quite close to the percolation threshold for site percolation in a 3D cubic lattice $[80,81]$.

Given the current structural knowledge on this system [49,55], it is difficult to assess the shape and individual size of the SC puddles. However, the nearly constant value of $\Delta T_{1}$ signifies that there is little change in the puddles' size distribution in regime II, at least before the interpuddle coupling sets in. This assumption is also supported by x-ray data [49]. The decrease of the ordered volume fraction $p$ with the cooling rate therefore must be related to an increase of the average interpuddle distance $d$. The typical value of $d$ can be crudely approximated assuming spherical puddles forming a simple cubic lattice: $p=\left[(4 \pi / 3)(L / 2)^{3}\right] /(L+d)^{3}$. For example, spheres of diameter $L=970 \mathrm{~nm}$ lead to $d=130 \mathrm{~nm}$ at $p=0.36$.

We here would like to comment on the distribution of the interpuddle distance $d$. Although we assumed in the above analysis that $d$ is the same for all puddles, we do not think that the actual distribution of $d$ is so sharp. Indeed, when this is the case, the final resistance drop would be much sharper, as observed in, e.g., Ref. [79]. In the other limit, when there is a wide distribution in $d$, the final drop would be much broader and illdefined [82]. Thus, the actual $d$ distribution in (TMTSF) ${ }_{2} \mathrm{ClO}_{4}$ is in a somewhat intermediate situation between the two limits. Nevertheless, we continue to use the constant- $d$ assumption, which is the simplest model to capture the physics behind, in the model calculation and analysis below.

Once superconductivity of individual puddles is established, further cooling enables interpuddle phase coherence via proximity effect through the normal metallic region in between, in a somewhat similar manner to the behavior of $\mathrm{Nb}$ islands on $\mathrm{Au}$ [79]. Macroscopic coherence is then reached when the first percolating path connects one end of the sample to the other. For phase coherence to be established between neighboring puddles, the thermal energy needs to be smaller than the proximity effect energy $[78,79,83]$ for the local SNS junction: $k_{\mathrm{B}} T \lesssim E_{\mathrm{J}}=\frac{\hbar I_{\mathrm{c}}}{2 e}$ where $E_{\mathrm{J}}$ is the Josephson energy, and $I_{\mathrm{c}}$ is the Josephson current running between neighboring puddles. Using the expression for $I_{\mathrm{c}}$ in SNS junctions given by the Usadel equations in the diluted limit $[83,84]$, one determines the temperature $T_{\mathrm{c} x}$ at which the macroscopic coherence is established:

$$
T_{\mathrm{c} \chi}=\frac{2 \hbar}{\pi e^{2} k_{\mathrm{B}}^{2}} \frac{\Delta_{0}^{2}\left(T_{\mathrm{c} \chi}\right)}{T_{\mathrm{c} \chi}} \frac{\mathcal{A}}{\rho_{\mathrm{N}}} \frac{1}{\xi_{\mathrm{N}}\left(T_{\mathrm{c} \chi}\right)} e^{-\left[d / \xi_{N}\left(T_{\mathrm{c} \chi}\right)\right]},
$$

where $\rho_{\mathrm{N}}$ is the resistivity of the normal metallic regions $(\mathrm{N})$ and should be close to $\rho_{\text {Max }}$ in our case, $\mathcal{A}$ is the averaged section of the junctions, $\Delta_{0}$ is the SC gap in each puddle:

$$
\Delta_{0}^{2}(T)=\frac{8 \pi^{2}}{7 \zeta(3)} k_{\mathrm{B}}^{2} T_{\mathrm{c} \rho}^{2}\left(1-\frac{T}{T_{\mathrm{c} \rho}}\right)
$$

for $T$ close to $T_{\mathrm{c} \rho}$, and $\zeta(3) \simeq 1.202$. In the Usadel equations $\xi_{\mathrm{N}}$, the normal-state coherence length in the $\mathrm{N}$ region, is determined by the dirty limit:

$$
\xi_{\mathrm{N}}(T)=\sqrt{\frac{1}{3} \frac{\hbar v_{\mathrm{F}}}{k_{\mathrm{B}} T} l} .
$$

Notice that the clean-limit coherence length $\xi_{\mathrm{N}}^{0}=\hbar v_{\mathrm{F}} / k T$ is much larger than $\xi_{\mathrm{N}}, d$, and $l \sim\left(\rho_{\mathrm{Min}} / \rho_{\mathrm{N}}\right) l_{0} \sim$ $\left(\rho_{\text {Min }} / \rho_{\text {Max }}\right) l_{0} \sim 190 \mathrm{~nm}$ in our case (for example, $\xi_{\mathrm{N}}^{0}=1400$ $\mathrm{nm}$ and $\xi_{\mathrm{N}}=290 \mathrm{~nm}$ at $1 \mathrm{~K}$ ) and thus the dirty-limit treatment is justified. Combining Eqs. (3)-(5), the relation between $T_{\mathrm{c} \rho}$ and $T_{\mathrm{c} \chi}$ can be expressed as

$$
T_{\mathrm{c} \chi}=\frac{\mathcal{C}}{\sqrt{T_{\mathrm{c} \chi}}}\left(1-\frac{T_{\mathrm{c} \chi}}{T_{\mathrm{c} \rho}}\right) e^{-\left[d(p) \sqrt{T_{\mathrm{c} \chi}} / \alpha\right]},
$$

where $\mathcal{C}$ and $\alpha$ are constants. This equation can be numerically solved to analyze the data.

Let us now compare this expression to our experimental results in Fig. 6. Assuming $d(p)$ for the model of spherical puddles forming a simple cubic lattice, the observed evolution of $T_{\mathrm{c} \chi}$ with $p$ is compatible with that evaluated from Eq. (6) (the red curve in Fig. 6). The fitting parameters obtained are $\alpha=$ $100 \mathrm{~nm} / \mathrm{K}^{1 / 2}$ and $\mathcal{C}=5.2 \mathrm{~K}^{3 / 2}$. The value of $\alpha$ in turns gives $\xi_{\mathrm{N}}=\alpha / \sqrt{T}=120 \mathrm{~nm}$ at $0.75 \mathrm{~K}$. This value is in reasonable agreement with what is expected in the disordered region [85]. The fitting result $\mathcal{C} \simeq 5.2 \mathrm{~K}^{3 / 2}$ also compares favorably with its theoretical estimate: assuming $\mathcal{A} \sim L^{2} \sim 9.4 \times 10^{-13} \mathrm{~m}^{2}$, $v_{\mathrm{F}}=1.8 \times 10^{5} \mathrm{~m} / \mathrm{s}, l=190 \mathrm{~nm}$, and $T_{\mathrm{c} \rho}=1.27 \mathrm{~K}$, we obtain $\mathcal{C}^{\text {theo }} \simeq 49 \mathrm{~K}^{3 / 2}$. Considering the randomness in the local distribution of puddles size, of their shape, as well as of the interpuddle distance that is not taken into consideration in our very crude model, $\mathcal{C}$ from experiment and theory agree reasonably with each other.

A second argument for this granular scenario comes from a previous specific-heat study performed on a sample cooled at the rate of $10 \mathrm{~K} / \mathrm{min}$ [51] (corresponding to $1-p=57 \%$; see Fig. 4). This study revealed a broad SC anomaly starting around $1.1 \mathrm{~K}$ and reaching a maximum around $0.85 \mathrm{~K}$. This temperature for the maximum specific heat matches $T_{\mathrm{c} \chi}$, whereas the onset of the broad transition roughly corresponds to the border between the light-blue and light-red regions in Fig. 6, namely the temperature domain where the pair condensation begins to be significant thermodynamically speaking. Furthermore, specific-heat data have revealed that the entropy involved in the SC pairing of this sample cooled at $10 \mathrm{~K} / \mathrm{min}$ [51] is only $50 \pm 10 \%$ of the total electronic specific heat. This feature is in fair agreement with the $\approx 40 \%$ value for the bulk SC volume fraction derived in Fig. 4. These agreements provide additional support for our scenario. As demonstrated above, the proximity effect together with percolation represent a reasonable approach for understanding the development of the superconducting state in regime II. 


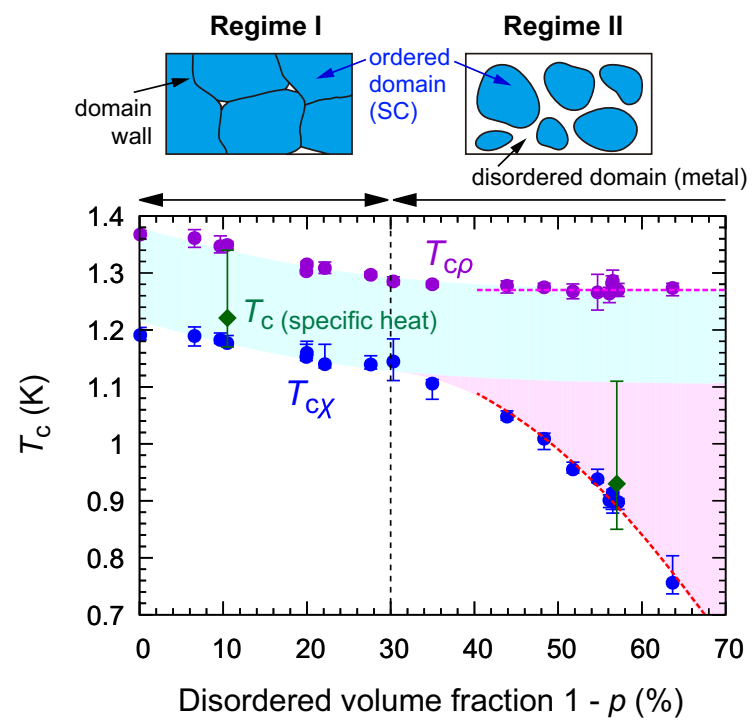

FIG. 6. Dependencies of $T_{\mathrm{c} \rho}$ (purple circles) and $T_{\mathrm{c} X}$ (blue circles) on the disordered volume fraction $1-p$ for sample no. 1 . Note that $T_{\mathrm{c} \chi}$ is practically equivalent to $T_{\mathrm{c}, \rho=0}$. For comparison, we plot $T_{\mathrm{c}}$ from the specific-heat study [51], with $1-p$ values estimated from the curves in Fig. 4. For these data, the upper error bar corresponds to the onset of the transition, and the lower error bar to the peak in the electronic specific heat. In the light-blue region, superconductivity develops within individual puddles. On the border between the light-blue and light-red regions, the proximity effect starts to induce SC coherence between puddles. The large scale phasecoherent superconductivity establishes progressively in the light-red region as a result of the interpuddle proximity effect coupling. The broken curves indicate the region used for the analysis based on the proximity effect: $T_{\mathrm{c} \rho}$ is assumed to be constant $(1.27 \mathrm{~K})$ as shown with the magenta curve and $T_{\mathrm{c} \chi}$ is calculated by solving Eq. (6). The red curve is obtained by fitting the theory to the $T_{\mathrm{c} x}$ data in the range $1-p>40 \%$. The resulting fitting parameters are $\mathcal{C}=5.2 \mathrm{~K}^{3 / 2}$ and $\alpha=100 \mathrm{~nm} / \mathrm{K}^{1 / 2}$.

Third, another support for our scenario is provided by the ac susceptibility data. Indeed, it is striking that a magnetic signal is only perceptible below $T_{\mathrm{c} \chi}$, when the measured resistivity is only a small fraction of that of the normal state. This signifies that the part of the sample contributing to the initial resistivity drop is, in all likelihood, formed of small isolated SC puddles which size ( $L \simeq 970 \mathrm{~nm}$ ) is smaller than or of the order of the SC penetration depth $(\lambda>1000 \mathrm{~nm}[86,87])$, so that these are invisible to ac susceptibility measurements. The dissipation is then due to currents circulating in the normal (disordered) regions [88].

As the temperature is lowered, in between $T_{\mathrm{c} \chi}$ and $T_{\mathrm{c} \rho}$, intergrain coupling starts, so that SC clusters form, resulting in a first percolating path at $T_{\mathrm{c}, \rho=0} \simeq T_{\mathrm{c} \chi}$, but also in a sizable shielded volume. Then $\Delta \chi_{\mathrm{ac}}^{\prime}$ begins to decrease, while $\Delta \chi_{\mathrm{ac}}^{\prime \prime}$ increases due to enhanced dissipation: as the SC volume expands, the current density in the remaining normal regions becomes also larger. More generally, as has been mentioned in the early studies of filamentary superconductors [76,89-91], the peak of $\Delta \chi_{\mathrm{ac}}^{\prime \prime}$ reflects a multiphases compound. Here, it arises from a balance between the normal and SC regions: the peak occurs at a temperature $T_{\text {peak }}$ when approximately half of the sample is shielded, as shown by the value of $\left.\Delta \chi_{\mathrm{ac}}^{\prime}\right|_{T_{\text {peak }}} \simeq-0.5$. This explains why, at higher cooling rates, $T_{\text {peak }}$ shifts to lower temperatures: at high cooling rates the initial density of SC puddles is lower, so that the coupling needs to be stronger, or the temperature lower, to achieve the same $\mathrm{SC}$ volume in the sample.

Finally, the $T \rightarrow 0$ limit of $\Delta \chi_{\mathrm{ac}}^{\prime \prime}$ is a measure of the dissipation and hence an indirect probe of the disordered volume fraction. This is illustrated in Fig. 5(c). As can be seen, $\Delta \chi_{\mathrm{ac}}^{\prime \prime}(T \rightarrow 0)$ is almost constant and close to 0 until $1-p \simeq 0.3$ [or equivalently, the cooling rate $\simeq 1 \mathrm{~K} / \mathrm{min}$; see Fig. 2(d)], before drastically increasing with the disordered volume fraction. The fact that $\Delta \chi_{\mathrm{ac}}^{\prime \prime}$ does not vanish even for $T \rightarrow 0$ provides yet another strong indication that normal regions persist in this limit.

Let us remark that this behavior is at variance with the behavior of cuprate high- $T_{\mathrm{c}}$ superconductors (HTSCs). Indeed, in these compounds, the two peaks observed in the measurements of $\Delta \chi_{\mathrm{ac}}^{\prime \prime}$ have been attributed to the onset of intra- and intergrain superconducting currents. Moreover, the initial drop in $\Delta \chi_{\mathrm{ac}}^{\prime}$ is steep in temperature, simultaneous with the resistive $T_{\mathrm{c}}$, and corresponds to the penetration of the field within each superconducting grain [92,93]. Finally, $\Delta \chi_{\mathrm{ac}}^{\prime}$ always goes to -1 in the low-temperature limit. In our case, however, the shielding is not always total, the drop in $\Delta \chi_{\mathrm{ac}}^{\prime}$ is broad in temperature, and only begins at $T_{\mathrm{c} \chi}$ where the resistivity measurements show that the percolation threshold is almost reached. In the present case, given the large size of the normal regions, the magnetic signal is more likely to be due to currents within the normal regions than to the penetration of currents in the SC regions, contrary to HTSCs.

\section{CONCLUSION}

Our precise study of the normal and superconducting properties of (TMTSF) $)_{2} \mathrm{ClO}_{4}$ by simultaneous transport and magnetic measurements, using a carefully controlled cooling procedure and covering cooling rates of four orders of magnitude, has revealed a crossover between a defect controlled $d$-wave and granular superconductivity in an inhomogeneous conductor with increasing cooling rate.

At cooling rates up to about $1 \mathrm{~K} / \mathrm{min}$, nonmagnetic disorder originates from small-volume clusters of disordered anions. The clusters are randomly distributed and act as local scattering centers. Superconductivity is suppressed by this disorder and the behavior of $T_{\mathrm{c}}$ versus the residual resistivity can be explained by the Abrikosov-Gorkov theory adapted to impure $d$ wave superconductors. Above $1 \mathrm{~K} / \mathrm{min}$ and up to $18 \mathrm{~K} / \mathrm{min}, T_{\mathrm{c}}$ for the resistivity onset remains independent of the cooling rate whereas $T_{\mathrm{c}}$ for the onset of the ac susceptibility signal keeps decreasing. In this fast-cooling-rate regime, the system behaves as a set of randomly distributed SC (anion-ordered) puddles embedded in a normal conducting background with disordered anions. The fraction of the sample volume comprising ordered anions has been derived using the theory for an effective medium composed of two regions with different residual resistivities. Zero resistance and diamagnetic shielding are achieved through proximity effect links between the bulk SC puddles. The volume fraction of bulk SC puddles is about 30\% smaller than the macroscopic screened sample volume measured by 
the real part of ac susceptibility. This feature provides an additional argument supporting the proximity effect picture in the fast-cooling regime. We emphasize that the case of (TMTSF) ${ }_{2} \mathrm{ClO}_{4}$ investigated in the present study is likely to be relevant for other $1 \mathrm{D}$ or $2 \mathrm{D}$ molecular superconductors when disorder arises either from molecular disorder or from the competition between other ground states [94-96]. Similarly, the granular behavior at high disorder concentration can occur in other unconventional superconductors, particularly when the disorder is introduced chemically and forms clusterlike structures. Thus the present study provides a guideline and an insight toward future investigation of impurity effects in a wide variety of unconventional superconductors.

When considering the competition between SC and SDW in the two materials (TMTSF) ${ }_{2} \mathrm{PF}_{6}$ and (TMTSF) ${ }_{2} \mathrm{ClO}_{4}$, it becomes clear that the corresponding driving mechanisms are different. For (TMTSF) ${ }_{2} \mathrm{PF}_{6}$ under pressure, from the high-pressure SC side, SDW develops as a consequence of a decrease of the unnesting term in the band structure (improving nesting in turn). Only in a narrow pressure regime close to the critical pressure can SC coexist with SDW albeit in distinct domains in the sample. In (TMTSF) $)_{2} \mathrm{ClO}_{4}$ on the other hand, the intermediate state consists of a mixture of anion-ordered puddles embedded in the anion-disordered background whose volume fraction depends on the cooling rate.

It would be particularly interesting to pursue this study to further understand how the electronic granularity arises from disorder. Comparing ac susceptibility measurementsin zero and finite magnetic field-in the different crystal orientations or STM measurements such as those performed on electronically inhomogeneous $\mathrm{NbN}$ thin films [97] could for example provide information on the size of the SC puddles and the mechanism responsible for their formation. Finally, let us note that the flexibility brought by the cooling procedure in tuning both the density and the dimensions of SC puddles and proximity effect bridges makes (TMTSF) ${ }_{2} \mathrm{ClO}_{4}$ a promising candidate in which more advanced investigations of mesoscopic superconductivity could be undertaken. One could, for instance, probe the quantum-critical metallic states that have been predicted to arise in such systems [98], study how grain superconductivity and proximity effect each contribute to the specific heat, as this issue has not yet been clearly settled [99], or study the localization-delocalization transition both in the three- and two-dimensional limits [100].

\section{ACKNOWLEDGMENTS}

S.Y. acknowledges Y. Maeno, Y. Sugimoto, T. Higuchi, and K. Ishida for experimental support and fruitful discussion. D.J. acknowledges fruitful exchanges with B. Castaing, who made him aware of the Landau effective conductor model used in this work, and with J. P. Pouget for discussions on X-ray data. We had several discussions with H. Bouchiat, D. Roditchev, and T. Cren on the topic of mesoscopic superconductivity. This work has been supported by Japan Society for the Promotion of Science (JSPS) Grant-in-Aids KAKENHI Grants No. JP26287078 and No. JP17H04848, as well as by JSPS Grant-in-Aids for Scientific Research on Innovative Areas "Topological Materials Science" (KAKENHI Grants No. JP15H05852 and No. JP15K21717).
[1] D. Jerome, A. Mazaud, M. Ribault, and K. Bechgaard, Superconductivity in a synthetic organic conductor (TMTSF) $)_{2} \mathrm{PF}_{6}$, J. Phys. Lett. (Paris) 41, L95 (1980).

[2] K. Bechgaard, K. Carneiro, M. Olsen, F. B. Rasmussen, and C. S. Jacobsen, Zero-Pressure Organic Superconductor: Di-(Tetramethyltetraselenafulvalenium)-Perchlorate [(TMTSF) ${ }_{2} \mathrm{ClO}_{4}$ ], Phys. Rev. Lett. 46, 852 (1981).

[3] J. Bednorz and K. A. Müller, Possible high $T_{\mathrm{c}}$ superconductivity in the Ba-La-Cu-O system, Z. Phys. B 64, 189 (1986).

[4] R. H. McKenzie, Similarities between organic and cuprate superconductors, Science 278, 820 (1997).

[5] K. Kanoda, Electron correlation, metal-insulator transition and superconductivity in quasi-2D organic systems, $(\mathrm{ET})_{2} \mathrm{X}$, Physica C 282-287, 299 (1997).

[6] S. Lefebvre, P. Wzietek, S. Brown, C. Bourbonnais, D. Jerome, C. Méziere, M. Fourmigué, and P. Batail, Mott Transition, Antiferromagnetism, and Unconventional Superconductivity in Layered Organic Superconductors, Phys. Rev. Lett. 85, 5420 (2000).

[7] Y. Kamihara, T. Watanabe, M. Hirano, and H. Hosono, Ironbased layered superconductor $\mathrm{La}\left[\mathrm{O}_{1-x} \mathrm{~F}_{x}\right] \mathrm{FeAs}(x=0.05-$ 0.12) with $T_{\mathrm{c}}=26 \mathrm{~K}$, J. Am. Chem. Soc. 130, 3296 (2008).

[8] Y. Maeno, H. Hashimoto, K. Yoshida, S. Nishizaki, T. Fujita, J. G. Bednorz, and F. Lichtenberg, Superconductivity in a layered perovskite without copper, Nature (London) 372, 532 (1994).

[9] A. P. Mackenzie, R. K. W. Haselwimmer, A. W. Tyler, G. G. Lonzarich, Y. Mori, S. Nishizaki, and Y. Maeno,
Extremely Strong Dependence of Superconductivity on Disorder in $\mathrm{Sr}_{2} \mathrm{RuO}_{4}$, Phys. Rev. Lett. 80, 161 (1998).

[10] P. J. Hirschfeld, Using gap symmetry and structure to reveal the pairing mechanism in Fe-based superconductors, C. R. Phys. 17, 197 (2016).

[11] K. Michaeli and L. Fu, Spin-Orbit Locking as a Protection Mechanism of the Odd-Parity Superconducting State against Disorder, Phys. Rev. Lett. 109, 187003 (2012).

[12] Y. Nagai, Robust superconductivity with nodes in the superconducting topological insulator $\mathrm{Cu}_{x} \mathrm{Bi}_{2} \mathrm{Se}_{3}$ : Zeeman orbital field and nonmagnetic impurities, Phys. Rev. B 91, 060502(R) (2015).

[13] Y. A. Bychkov, L. P. Gorkov, and I. Dzyaloshinskii, Possibility of superconductivity type phenomena in a one-dimensional system, Zh. Eksp. Teor. Fiz. 50, 738 (1966) [Sov. Phys. JETP 23, 489 (1966)].

[14] V. J. Emery, Theory of the one-dimensional electron gas, in Highly Conducting One-Dimensional Solids, edited by J. T. Devreese, R. E. Evrard, and V. E. van Doren (Plenum, New York, 1979), p. 247.

[15] J. Sólyom, The Fermi gas model of one-dimensional conductors, Adv. Phys. 28, 201 (1979).

[16] D. Jérome and H. Schulz, Organic conductors and superconductors, Adv. Phys. 31, 299 (1982).

[17] T. Ishiguro, K. Yamaji, and G. Saito, Organic Superconductors (Springer, Berlin, 1998). 
[18] T. Giamarchi, Quantum Physics in One-Dimension (Clarendon, Oxford, 2004).

[19] K. Yamaji, First-order phase transition boundary between superconducting and SDW phases in the bechgaard salts, J. Phys. Soc. Jpn. 52, 1361 (1983).

[20] G. Montambaux, Metal-spin-density-wave transition in a quasi-one-dimensional conductor: Pressure and magnetic field effects, Phys. Rev. B 38, 4788 (1988).

[21] M. Takigawa, H. Yasuoka, and G. Saito, Proton spin relaxation in the superconducting state of (TMTSF) ${ }_{2} \mathrm{ClO}_{4}$, J. Phys. Soc. Jpn. 56, 873 (1987).

[22] S. Belin and K. Behnia, Thermal Conductivity of Superconducting (TMTSF) ${ }_{2} \mathrm{ClO}_{4}$ : Evidence for a Nodeless Gap, Phys. Rev. Lett. 79, 2125 (1997).

[23] N. Joo, P. Auban-Senzier, C. Pasquier, P. Monod, D. Jérome, and K. Bechgaard, Suppression of superconductivity by non-magnetic disorder in the organic superconductor $(\mathrm{TMTSF})_{2}\left(\mathrm{ClO}_{4}\right)_{(1-x)}\left(\mathrm{ReO}_{4}\right)_{x}$, Eur. Phys. J. B 40, 43 (2004).

[24] N. Joo, P. Auban-Senzier, C. Pasquier, D. Jérome, and K. Bechgaard, Impurity-controlled superconductivity/spin density wave interplay in the organic superconductor: (TMTSF) ${ }_{2} \mathrm{ClO}_{4}$, Europhys. Lett. 72, 645 (2005).

[25] J. Shinagawa, Y. Kurosaki, F. Zhang, C. Parker, S. E. Brown, D. Jérome, K. Bechgaard, and J. B. Christensen, Superconducting State of the Organic Conductor (TMTSF) ${ }_{2} \mathrm{ClO}_{4}$, Phys. Rev. Lett. 98, 147002 (2007).

[26] S. Yonezawa, Y. Maeno, K. Bechgaard, and D. Jérome, Nodal superconducting order parameter and thermodynamic phase diagram of (TMTSF) $)_{2} \mathrm{ClO}_{4}$, Phys. Rev. B 85, 140502(R) (2012).

[27] F. L. Pratt, T. Lancaster, S. J. Blundell, and C. Baines, LowField Superconducting Phase of (TMTSF) ${ }_{2} \mathrm{ClO}_{4}$, Phys. Rev. Lett. 110, 107005 (2013).

[28] D. Jerome and S. Yonezawa, Novel superconducting phenomena in quasi-one-dimensional Bechgaard salts, C. R. Phys. 17, 357 (2016).

[29] V. J. Emery, The mechanisms of organic superconductivity, Synth. Met. 13, 21 (1986).

[30] Y. Hasegawa and H. Fukuyama, A theory of phase transitions in quasi-one-dimensional electrons, J. Phys. Soc. Jpn. 55, 3978 (1986).

[31] C. Bourbonnais and L. G. Caron, The role of kinetic interchain coupling in Quasi-1D conductors, Physica B+C 143, 450 (1986).

[32] Y. Hasegawa and H. Fukuyama, NMR Relaxation Time of the anisotropic superconducting state in quasi-one-dimensional systems, J. Phys. Soc. Jpn. 56, 877 (1987).

[33] C. Bourbonnais, L. Caron, F. Creuzet, and D. Jérome, Mechanisms for antiferromagnetism and superconductivity in the bechgaard salts, J. Phys. Colloq. 49, C8 (1988).

[34] A. P. Schnyder and P. M. R. Brydon, Topological surface states in nodal superconductors, J. Phys.: Condens. Matter 27, 243201 (2015).

[35] S. Yonezawa, Bulk topological superconductors, AAPPS Bull. 26, 3 (2016).

[36] S. Bouffard, M. Ribault, R. Brusetti, D. Jérome, and K. Bechgaard, Low-temperature metallic state and superconductivity in quasi-one-dimensional organic conductors: pressure and irradiation investigations, J. Phys. C 15, 2951 (1982).
[37] M. Y. Choi, P. M. Chaikin, S. Z. Huang, P. Haen, E. M. Engler, and R. L. Greene, Effect of radiation damage on the metal-insulator transition and low-temperature transport in the tetramethyltetraselenofulvalinium $\mathrm{PF}_{6}$ salt $\left[(\mathrm{TMTSF})_{2} \mathrm{PF}_{6}\right.$, Phys. Rev. B 25, 6208 (1982).

[38] A. A. Abrikosov, Superconductivity in a quasi-onedimensional metal with impurities, J. Low Temp. Phys. 53, 359 (1983).

[39] L. Zuppirolli, in Low Dimensional Conductors and Superconductors, edited by D. Jerome and L. G. Caron (Plenum, New York, 1987), p. 307.

[40] M. Sanquer and S. Bouffard, ESR properties of irradiated (TMTSF) ${ }_{2} \mathrm{ClO}_{4}$, Mol. Cryst. Liq. Cryst. 119, 147 (1985).

[41] S. Tomić, D. Jérôme, D. Mailly, M. Ribault, and K. Bechgaard, Influence of the disorder potential of the anions on the ground state of the organic alloy $(\mathrm{TMTSF})_{2}\left(\mathrm{ClO}_{4}\right)_{1-x}\left(\mathrm{ReO}_{4}\right)_{x}$, J. Phys. Colloq. 44, C3-1075 (1983).

[42] P. Garoche, R. Brusetti, D. Jerome, and K. Bechgaard, Specific heat measurements of organic superconductivity in (TMTSF) ${ }_{2} \mathrm{ClO}_{4}$, J. Phys. Lett. (Paris) 43, L147 (1982).

[43] H. Schwenk, K. Neumaier, K. Andres, F. Wudl, and E. AharonShalom, Meissner anisotropy in deuterated (TMTSF) ${ }_{2} \mathrm{ClO}_{4}$, Mol. Cryst. Liq. Cryst. 79, 633 (1982).

[44] S. Tomić, D. Jérome, P. Monod, and K. Bechgaard, EPR and electrical conductivity of the organic superconductor di-tetramethyltetraselenafulvalenium-perchlorate, (TMTSF $)_{2} \mathrm{ClO}_{4}$ and a metastable magnetic state obtained by fast cooling, J. Phys. Lett. 43, 839 (1982).

[45] T. Ishiguro, K. Murata, K. Kajimura, N. Kinoshita, H. Tokumoto, M. Tokumoto, T. Ukachi, H. Anzai, and G. Saito, Superconductivity and metal-nonmetal transitions in (TMTSF) $)_{2} \mathrm{ClO}_{4}$, J. Phys. Colloq. 44, C3-831 (1983).

[46] T. Takahashi, D. Jérome, and K. Bechgaard, Observation of a magnetic state in the organic superconductor (TMTSF) ${ }_{2} \mathrm{ClO}_{4}$ : influence of the cooling rate, J. Phys. Lett. 43, L565 (1982).

[47] J. P. Pouget, G. Shirane, K. Bechgaard, and J. M. Fabre, X-ray evidence of a structural phase transition in di-tetramethyltetraselenafulvalenium perchlorate [(TMTSF $\left.)_{2} \mathrm{ClO}_{4}\right]$, pristine and slightly doped, Phys. Rev. B 27, 5203 (1983).

[48] D. Gubser, W. Fuller, T. Poehler, J. Stokes, D. Cowan, M. Lee, and A. Bloch, Resistive and magnetic susceptibility transitions in superconducting (TMTSF) ${ }_{2} \mathrm{ClO}_{4}$, Mol. Cryst. Liq. Cryst. 79, 581 (1982).

[49] J. P. Pouget, Structural aspects of the bechgaard and fabre salts: An update, Crystals 2, 466 (2012).

[50] H. Schwenk, K. Andres, and F. Wudl, Resistivity of the organic superconductor ditetramethyltetraselenafulvalenium perchlorate, $(\mathrm{TMTSF})_{2} \mathrm{ClO}_{4}$, in its relaxed, quenched, and intermediate state, Phys. Rev. B 29, 500 (1984).

[51] P. Garoche, R. Brusetti, and K. Bechgaard, Influence of the Cooling Rate on the Superconducting Properties of the Organic Solid Di-TetramethyltetraselenafulvaleniumPerchlorate, (TMTSF) $)_{2} \mathrm{ClO}_{4}$, Phys. Rev. Lett. 49, 1346 (1982).

[52] N. Matsunaga, A. Ishikawa, A. Hoshikawa, K. Nomura, S. Takasaki, J. Yamada, S. Nakatsuji, and H. Anzai, Anion disorder and two-dimensionality in the superconducting and SDW states of (TMTSF) $)_{2} \mathrm{ClO}_{4}$, J. Low Temp. Phys. 117, 1735 (1999). 
[53] S. Kagoshima, T. Yasunaga, T. Ishiguro, H. Anzai, and G. Saito, Quenching effect of the anion ordering in the organic superconductor (TMTSF) ${ }_{2} \mathrm{ClO}_{4}$ : An X-ray study, Solid State Commun. 46, 867 (1983).

[54] R. Moret, J. P. Pouget, R. Comès, and K. Bechgaard, $\mathrm{X}$-ray study of the anion ordering transition in di(tetramethyltetraselenafulvalen)-ium perchlorate (TMTSF) ${ }_{2} \mathrm{ClO}_{4}$ : quenching and irradiation effects, J. Phys. (France) 46, 1521 (1985).

[55] J.-P. Pouget, S. Kagoshima, T. Tamegai, Y. Nogami, K. Kubo, T. Nakajima, and K. Bechgaard, High resolution x-ray scattering study of the anion ordering phase transition of (TMTSF) ${ }_{2} \mathrm{ClO}_{4}$, J. Phys. Soc. Jpn. 59, 2036 (1990).

[56] The reason why we chose to measure $\rho_{c^{*}}$ is that the reproducibility of the data among different runs is especially important for the present investigation: Measurements of transport along the $a$ or $b$ axes are often perturbed by sample cracks occurring during cooling. In contrast, $\rho_{c^{*}}$ is known to be less influenced by such cracks even after multiple cooling processes [101]. In addition, magnetoresistance measurements [101] have shown that the band theory should apply below $10 \mathrm{~K}$ as also supported by the existence of a Drude edge along the $c^{*}$ axis [102].

[57] S. Haddad, S. Charfi-Kaddour, and J. P. Pouget, Inhomogeneous superconductivity in organic conductors: the role of disorder and magnetic field, J. Phys.: Condens. Matter 23, 464205 (2011).

[58] See Supplemental Material at http://link.aps.org/supplemental/ 10.1103/PhysRevB.97.014521 for details of experimental setups and procedures, and for results of sample no. 2.

[59] S. Yonezawa, T. Higuchi, Y. Sugimoto, C. Sow, and Y. Maeno, Compact AC susceptometer for fast sample characterization down to 0.1 K, Rev. Sci. Instrum. 86, 093903 (2015).

[60] P. Auban-Senzier, C. R. Pasquier, D. Jérome, and K. Bechgaard, Fluctuating spin density wave conduction in (TMTSF) ${ }_{2} X$ organic superconductors, Europhys. Lett. 94, 17002 (2011).

[61] N. Doiron-Leyraud, P. Auban-Senzier, S. René de Cotret, C. Bourbonnais, D. Jérome, K. Bechgaard, and L. Taillefer, Correlation between linear resistivity and $\mathrm{Tc}$ in the Bechgaard salts and the pnictide superconductor $\mathrm{Ba}\left(\mathrm{Fe}_{1-x} \mathrm{Co}_{x}\right)_{2} \mathrm{As}_{2}$, Phys. Rev. B 80, 214531 (2009).

[62] N. Doiron-Leyraud, P. Auban-Senzier, S. René de Cotret, C. Bourbonnais, D. Jérome, K. Bechgaard, and L. Taillefer, Linear-T scattering and pairing from antiferromagnetic fluctuations in the (TMTSF) $)_{2} X$ organic superconductors, Eur. Phys. J. B 78, 23 (2010).

[63] A. Sedeki, D. Bergeron, and C. Bourbonnais, Extended quantum criticality of low-dimensional superconductors near a spindensity-wave instability, Phys. Rev. B 85, 165129 (2012).

[64] M. Creyssels, C. Laroche, E. Falcon, and B. Castaing, Pressure dependence of the electrical transport in granular materials, Eur. Phys. J. E 40, 56 (2017).

[65] L. D. Landau and E. M. Lifshitz, Electrodynamical of Continous Media (Pergamon, Oxford, 1960), p. 45.

[66] It is intended that the model which is used here establishes a relationship between the cooling rate and the volume fraction in order to link experimental results to the theory of percolation to be mentioned later. It may be oversimplified as it neglects the weak volume dependence of the residual resistivity of ordered regions in regime I discussed in Sec. IV B.
[67] Y. Sun and K. Maki, Impurity effects in $d$-wave superconductors, Phys. Rev. B 51, 6059 (1995).

[68] Y. Suzumura and H. J. Schulz, Thermodynamic properties of impure anisotropic quasi-one-dimensional superconductors, Phys. Rev. B 39, 11398 (1989).

[69] A. A. Abrikosov and L. P. Gorkov, Contribution to the theory of superconducting alloys with paramagnetic impurities, Zh. Eksp. Teor. Fiz. 39, 1781 (1960) [Sov. Phys. JETP 12, 1243 (1961)].

[70] P. Alemany, J.-P. Pouget, and E. Canadell, Electronic structure and anion ordering in (TMTSF) ${ }_{2} \mathrm{ClO}_{4}$ and (TMTSF) ${ }_{2} \mathrm{NO}_{3}$ : A first-principles study, Phys. Rev. B 89, 155124 (2014).

[71] A criterion may be the width of the Fermi surface to be sufficiently small compared to the $k_{\mathrm{F}}$ separation of the ordered electronic structure, i.e., the number of unit cells building up a disordered cluster should thus exceed at least $4 t_{\|} / \Delta_{\mathrm{AO}} \approx 50$ since $4 t_{\|}=0.8 \mathrm{eV} \mathrm{[16]} \mathrm{and} \Delta_{\mathrm{AO}}=14 \mathrm{meV}$ [70]. This volume condition requires a finite volume related to the scattering centers even in the AG regime.

[72] According to the transport data displaying $T_{\mathrm{c}}$ vs $\rho_{c^{*} 0}$, the critical scattering rate, $\tau_{\mathrm{c}}^{-1}=0.88 k_{\mathrm{B}} T_{\mathrm{c} 0}$ and $T_{\mathrm{c} 0}=1.5 \mathrm{~K}$, provides $\tau_{\mathrm{c}}=5.8 \times 10^{-12} \mathrm{sec}$ leading in turn to $\tau_{0}=4.6 \times$ $10^{-11} \mathrm{sec}$ for the lowest cooling rate. This latter elastic lifetime is admittedly longer than values derived from the analysis of angular magnetoresistance (AMRO) experiments in (TMTSF) ${ }_{2} \mathrm{ClO}_{4}$ [103]. Such a situation has already been encountered and commented on in a previous study [104]. It has been attributed to the anisotropy in impurity scattering which may weaken the suppression of $T_{\mathrm{c}}$ while a $k$ averaged shorter $\tau$ should appear fully in the theory of angular magnetoresistance.

[73] In this rest analysis, we use the longitudinal scattering rate $1 / \tau_{0}$ as a measure for the elastic disorder governing $T_{\mathrm{c}}$ although the corresponding mean free path is known to be highly anisotropic [101]. Previous comparative studies of resistivities along the $a$ and $c^{*}$ directions have shown $[101,105]$ that both resistivities exhibit similar temperature dependencies up to $30 \mathrm{~K}$ in (TMTSF $)_{2} \mathrm{ClO}_{4}$. This behavior provides an additional confirmation for the existence of a $3 \mathrm{D}$ coherent regime at low temperature (albeit the non-Fermi-liquid-like temperature dependence of the resistivity).

[74] S. Tomić, J. R. Cooper, D. Jérome, and K. Bechgaard, NonOhmic Electrical Transport in the Spin-Density-Wave State of Tetramethyltetraselenafulvalinium Nitrate, (TMTSF) ${ }_{2} \mathrm{NO}_{3}$, Phys. Rev. Lett. 62, 462 (1989).

[75] K. Murata, M. Tokumoto, H. Anzai, K. Kajimura, and T. Ishiguro, Upper critical field of the anisotropic organic superconductors, (TMTSF) ${ }_{2} \mathrm{CIO}_{4}$, Jpn. J. Appl. Phys. 26, 1367 (1987).

[76] Y. Oda, H. Takenaka, H. Nagano, and I. Nakada, The Meissner effect of polysulfur nitride, $(\mathrm{SN})_{x}$, Solid State Commun. 32, 659 (1979).

[77] G. Deutscher, O. Entin-Wohlman, S. Fishman, and Y. Shapira, Percolation description of granular superconductors, Phys. Rev. B 21, 5041 (1980).

[78] C. J. Lobb, D. W. Abraham, and M. Tinkham, Theoretical interpretation of resistive transition data from arrays of superconducting weak links, Phys. Rev. B 27, 150 (1983).

[79] S. Eley, S. Gopalakrishnan, P. M. Goldbart, and N. Mason, Approaching zero-temperature metallic states in mesoscopic 
superconductor-normal-superconductor arrays, Nat. Phys. 8, 59 (2012).

[80] S. Kirkpatrick, Percolation and conduction, Rev. Mod. Phys. 45, 574 (1973).

[81] D. Stauffer and A. Aharony, Introduction to Percolation Theory (Taylor and Francis, London, 1994).

[82] A. Allain, Z. Han, and V. Bouchiat, Electrical control of the superconducting-to-insulating transition in graphene-metal hybrids, Nat. Mater. 11, 590 (2012).

[83] K. K. Likharev, Superconducting weak links, Rev. Mod. Phys. 51, 101 (1979).

[84] M. Tinkham, Introduction to Superconductivity (Dover Publications, New York, 2004).

[85] In disordered regions, $l^{a} \simeq 162 \mathrm{~nm}, l^{b} \simeq 16 \mathrm{~nm}$, and $l^{c} \simeq 8 \mathrm{~nm}$, so that $\xi_{\mathrm{N}}^{a} \simeq 380 \mathrm{~nm}, \xi_{\mathrm{N}}^{b} \simeq 31 \mathrm{~nm}$, and $\xi_{\mathrm{N}}^{c} \simeq 5 \mathrm{~nm}$. Although there most probably is an anisotropy in the proximity effect between neighboring superconducting islands, the overall value for $\xi_{\mathrm{N}}$ would in all likelihood be an average of $\xi_{\mathrm{N}}^{a}, \xi_{\mathrm{N}}^{b}$, and $\xi_{\mathrm{N}}^{c}$.

[86] L. P. Le, A. Keren, G. M. Luke, B. J. Sternlieb, W. D. Wu, Y. J. Uemura, J. H. Brewer, T. M. Riseman, R. V. Upasani, L. Y. Chiang, W. Kang, P. M. Chaikin, T. Csiba, and G. Grüner, Muon-spin-rotation and relaxation studies in (TMTSF) $)_{2} X$ compounds, Phys. Rev. B 48, 7284 (1993).

[87] A. J. Greer, D. Harshman, W. Kossler, A. Goonewardene, D. Williams, E. Koster, W. Kang, R. Kleiman, and R. Haddon, Muon spin rotation study of the (TMTSF) ${ }_{2} \mathrm{ClO}_{4}$ system, Physica C 400, 59 (2003).

[88] The sample dimensions are smaller than the skin depth $\delta$ at the considered frequencies: $\delta \simeq 2 \mathrm{~cm}$.

[89] E. Maxwell and M. Strongin, Filamentary Structure in Superconductors, Phys. Rev. Lett. 10, 212 (1963).

[90] M. Strongin, E. Maxwell, and T. B. Reed, Ac susceptibility measurements on transition metal superconductors containing rare earth and ferromagnetic metal solutes, Rev. Mod. Phys. 36, 164 (1964).

[91] Y. Oda, H. Takenaka, H. Nagano, and I. Nakada, A.C. susceptibility of polysulfur nitride $(\mathrm{SN})_{x}$, Solid State Commun. 35, 887 (1980).

[92] K.-H. Müller, AC susceptibility of high temperature superconductors in a critical state model, Physica C 159, 717 (1989).

[93] M. Nikolo, Superconductivity: A guide to alternating current susceptibility measurements and alternating current susceptometer design, Am. J. Phys. 63, 57 (1995).

[94] S. Senoussi and F. Pesty, Signatures of granular superconductivity and Josephson effects in macroscopic measurements: the case of new superconductors, Iran. J. Phys. Res. 6, 226 (2006).
[95] J. Müller, J. Brandenburg, and J. A. Schlueter, MagneticField Induced Crossover of Superconducting Percolation Regimes in the Layered Organic Mott System $\kappa$-(BEDT-TTF $)_{2} \mathrm{Cu}\left[\mathrm{N}(\mathrm{CN})_{2}\right] \mathrm{Cl}$, Phys. Rev. Lett. 102, 047004 (2009).

[96] B. J. Powell and R. H. McKenzie, Dependence of the superconducting transition temperature of organic molecular crystals on intrinsically nonmagnetic disorder: A signature of either unconventional superconductivity or the atypical formation of magnetic moments, Phys. Rev. B 69, 024519 (2004).

[97] C. Carbillet, S. Caprara, M. Grilli, C. Brun, T. Cren, F. Debontridder, B. Vignolle, W. Tabis, D. Demaille, L. Largeau, K. Ilin, M. Siegel, D. Roditchev, and B. Leridon, Confinement of superconducting fluctuations due to emergent electronic inhomogeneities, Phys. Rev. B 93, 144509 (2016).

[98] B. Spivak, P. Oreto, and S. A. Kivelson, Theory of quantum metal to superconductor transitions in highly conducting systems, Phys. Rev. B 77, 214523 (2008).

[99] S. Poran, T. Nguyen-Duc, A. Auerbach, N. Dupuis, A. Frydman, and O. Bourgeois, Quantum criticality at the superconductor-insulator transition revealed by specific heat measurements, Nat. Commun. 8, 14464 (2017).

[100] V. F. Gantmakher and V. T. Dolgopolov, Localized-delocalized electron quantum phase transitions, Phys. Usp. 51, 3 (2008).

[101] J. R. Cooper, L. Forró, B. Korin-Hamzić, K. Bechgaard, and A. Moradpour, Magnetoresistance of the organic conducting tetramethyltetraselenafulvalene salts (TMTSF) ${ }_{2} \mathrm{ClO}_{4}$ and (TMTSF) $)_{2} \mathrm{PF}_{6}$ : Search for the coherent-diffusive transition or localization effects with increasing temperature, Phys. Rev. B 33, 6810 (1986).

[102] W. Henderson, V. Vescoli, P. Tran, L. Degiorgi, and G. Grüner, Anisotropic electrodynamics of low dimensional metals: Optical studies of (TMTSF) ${ }_{2} \mathrm{ClO}_{4}$, Eur. Phys. J. B 11, 365 (1999).

[103] S. Sugawara, T. Ueno, Y. Kawasugi, N. Tajima, Y. Nishio, and K. Kajita, Out-of-plane resistance under transverse magnetic field in quasi-one-dimensional layered metals, J. Phys. Soc. Jpn. 75, 053704 (2006).

[104] S. Yonezawa, S. Kusaba, Y. Maeno, P. Auban-Senzier, C. Pasquier, and D. Jérome, Magnetic-field variations of the pair-breaking effects of superconductivity in (TMTSF) ${ }_{2} \mathrm{ClO}_{4}$, J. Phys. Soc. Jpn. 77, 054712 (2008).

[105] P. Auban-Senzier, D. Jérome, N. Doiron-Leyraud, S. René de Cotret, A. Sedeki, C. Bourbonnais, L. Taillefer, P. Alemany, E. Canadell, and K. Bechgaard, The metallic transport of (TMTSF $)_{2} X$ organic conductors close to the superconducting phase, J. Phys.: Condens. Matter 23, 345702 (2011). 